\title{
FRECUENCIA E INTENSIDAD DEL VIENTO EN ZARAGOZA
}

\author{
$M^{3}$ Luz HERNANDEZ NAVARRO \\ Universidad de Zaragoza.
}

\begin{abstract}
RESUMEN: Este artículo estudia la frecuencia e intensidad del viento en el observatorio de Zaragoza "Aeropuerto", en el periodo 1957-86. Analiza la velocidad media mensual, la velocidad mensual según las direcciones de la rosa de los vientos, según intervalos de velocidad, el porcentaje de calmas, las rachas máximas observadas en ese periodo y la velocidad media diaria en el periodo 1982-86.
\end{abstract}

\begin{abstract}
This article studies the frequency and wind intensity al Zaragoza "Aeropuerto" along the period 1957-86. It analyzes the mensual mean speed, the mean speed according to the compass rose, according to speed intervals, the percentage of calms, the maxim gust of wind and the daily mean speed along the period 1982-86.
\end{abstract}

Palabras clave: Velocidad viento, frecuencia, intensidad, Zaragoza, calmas, rachas máxima, viento diurno y nocturno.

Sumario: Introducción.- Material y métodos.- Resultados.- Conclusiones.- Bibliografía.

\section{INTRODUCCION}

El viento es un elemento de particular importancia en el Valle del Ebro, por la frecuencia con que se observa. Su encuadramiento entre dos macizos montañosos de considerable altitud forma un pasillo de dirección casi obligada para el viento, que describe una trayectoria Noroeste-Sureste o al contrario (cierzo o bochorno, respectivamente), la mayor parte de las ocasiones.

A pesar de su importancia climática y socio-económica son pocos los trabajos publicados sobre su dinámica. De referencia obligada es el trabajo de BIEL LUCEA 
(1952) El viento en Zaragoza, publicado por el entonces Servicio Meteorológico Nacional.

EL objeto de este pequeño trabajo es contribuir al conocimiento de este meteoro, su frecuencia de aparición y su intensidad o, lo que es lo mismo, su velocidad.

\section{MATERIAL Y METODOS}

Hemos utilizado los datos de viento del observatorio de Zaragoza "Aeropuerto", del Instituto Nacional de Meteorología, para el periodo 1957-86, cuyas coordenadas son: longitud: $41^{2} 43^{\prime} 43^{\prime \prime} \mathrm{N}$

latitud: $1^{\circ} 00^{\prime} 39^{\prime \prime} \mathrm{W}$

altitud: $257 \mathrm{~m}$.

para analizar:

-la velocidad media mensual del viento, en $\mathrm{km} / \mathrm{h}$, a $20 \mathrm{~m}$. de altura.

-la velocidad media mensual, a $20 \mathrm{~m}$. según la dirección de la rosa de los vientos.

-la velocidad del viento por intervalos de intensidad, en $\%$, a $20 \mathrm{~m}$. El periodo de estudio para el análisis es de 1974-86, ya que es a partir de esta fecha cuando el Instituto Nacional de Meteorología fijo estos intervalos de intensidad.

-las rachas máximas registradas, a $20 \mathrm{~m}$., con el análisis de alguna situación de tiempo representativa.

-la velocidad del viento diaria a $0,7,13$ y $18 \mathrm{~h}$., para el periodo $1982-86$, y la relación viento diurno-viento nocturno.

\section{RESULTADOS}

El viento sopla de forma continuada e intensa en Zaragoza. Su velocidad media anual es de $19 \mathrm{~km} / \mathrm{h}$, a los $20 \mathrm{~m}$. de altura, en el periodo 1957-86. Vemos en la figura $\mathrm{n}^{\mathrm{Q}}$ 1 cómo los meses de primavera e invierno son aquéllos en que el viento es más veloz (22 $\mathrm{km} / \mathrm{h}$. en abril). Es interesante resaltar el hecho de que la velocidad del viento sea también alta en verano $(19,4 \mathrm{~km} / \mathrm{h}$. en julio), lo que unido a las altas temperaturas estivales multiplica las necesidades hídricas de las plantas, y refuerza los efectos de la sequía veraniega. 
Según su dirección el viento sopla con más velocidad cuando es flujo del tercer cuadrante, direcciónes Oeste y Noroeste particularmante (figura $\mathrm{n}^{2} 2$ ). El bien conocido cierzo se presenta con una velocidad superior a los $20 \mathrm{~km} / \mathrm{h}$. durante todo el año, y rozando los $30 \mathrm{~km} / \mathrm{h}$. desde noviembre hasta abril. En el resto de direcciones se observan velocidades más moderadas, que ya no suelen sobrepasar los $15 \mathrm{~km} / \mathrm{h}$.

Las figuras $n^{2} 3$ nos muestra el bajo porcentaje de calmas y la figura $n^{0} 4$ las veces en que se observa viento según intervalos de intensidad, donde podemos comprobar el elevado tanto por ciento de ocasiones donde la velocidad del viento es alta.

Fig.1. Velocidad media del viento, en $\mathrm{km} / \mathrm{h}$

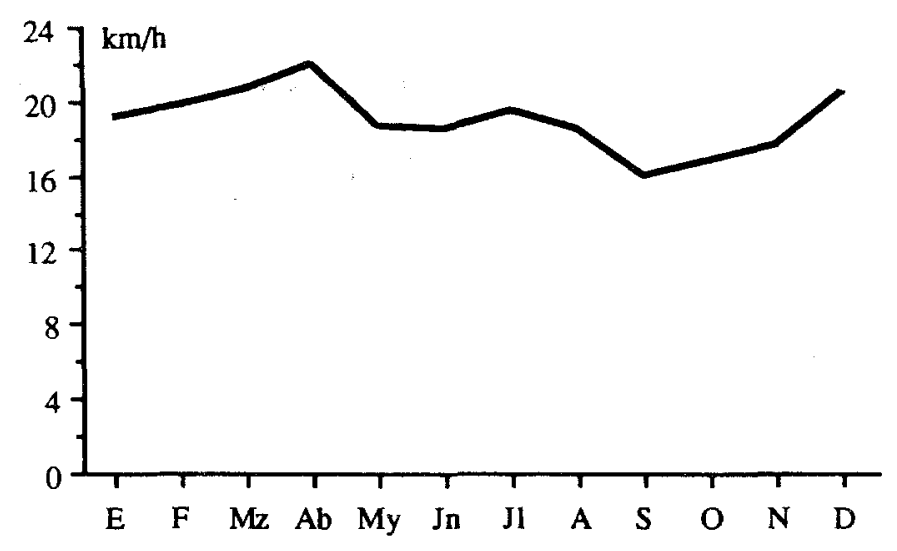

Fuente: Instituto Nacional de Meteorología Elaboración propia

Fig.3. Calmas, en \%

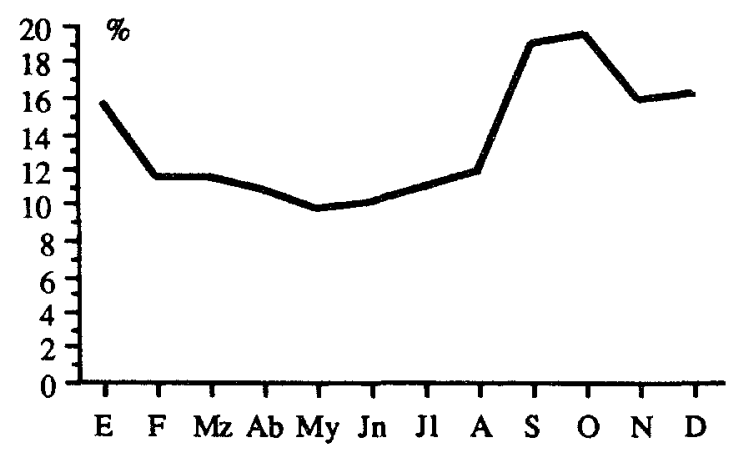

Fuente: Instituto Nacional de Meteorología. Elaboración propia 
Fig. 2. Velocidad del viento según dirección
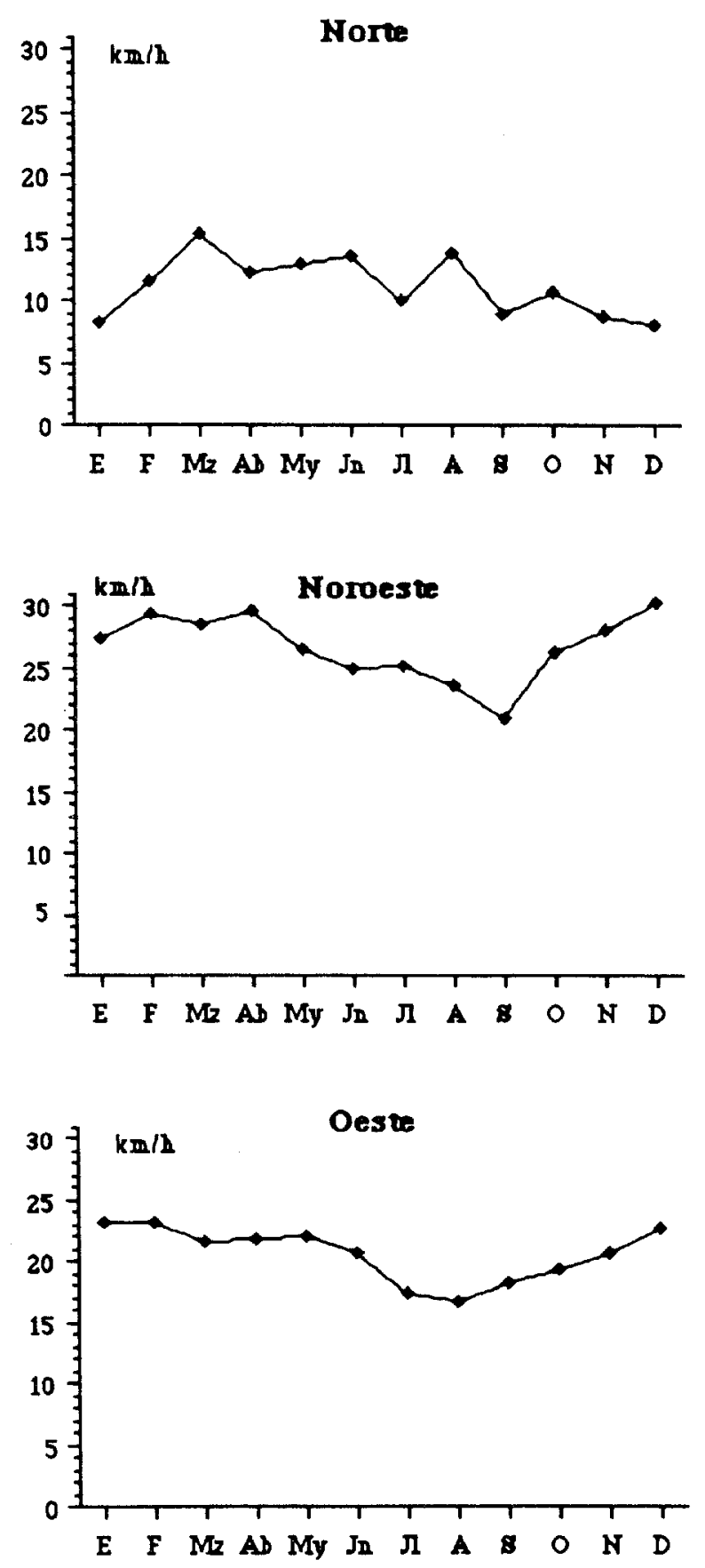

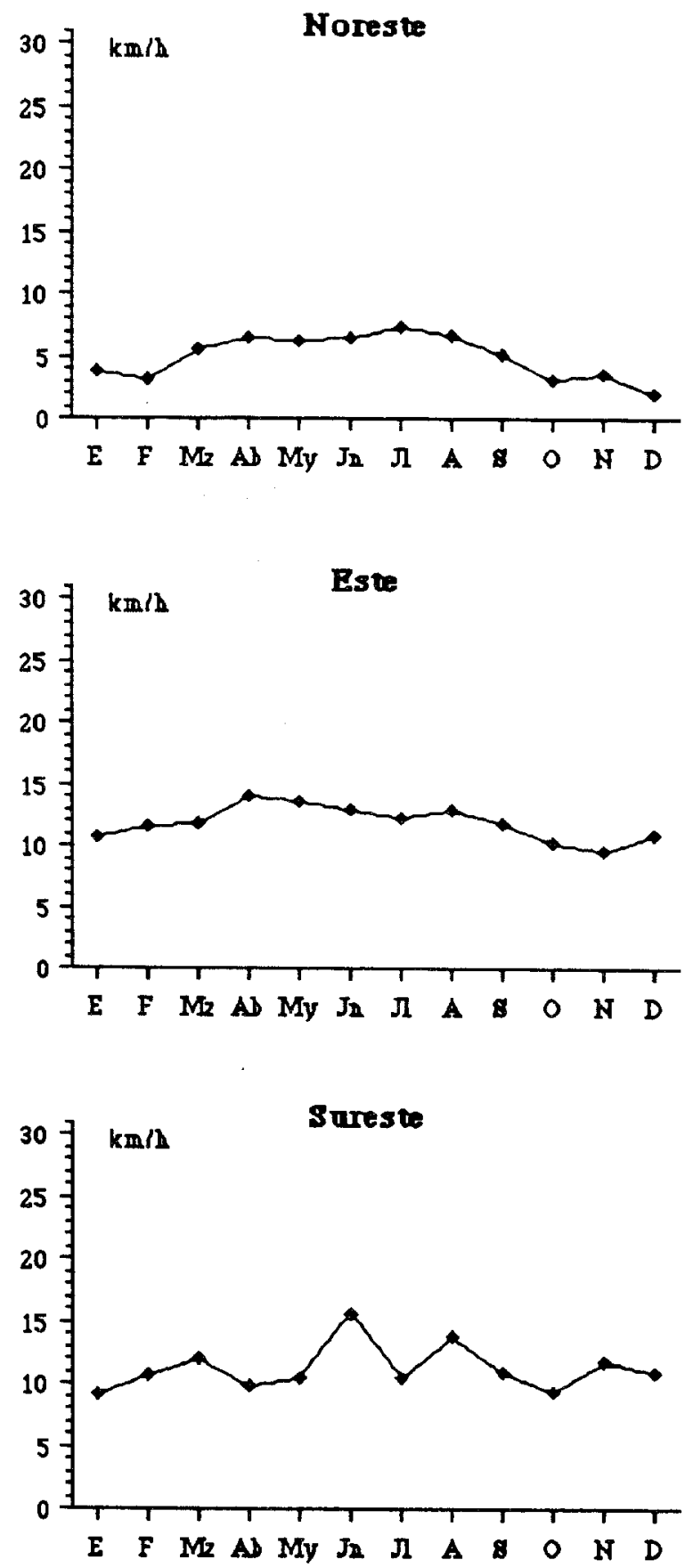

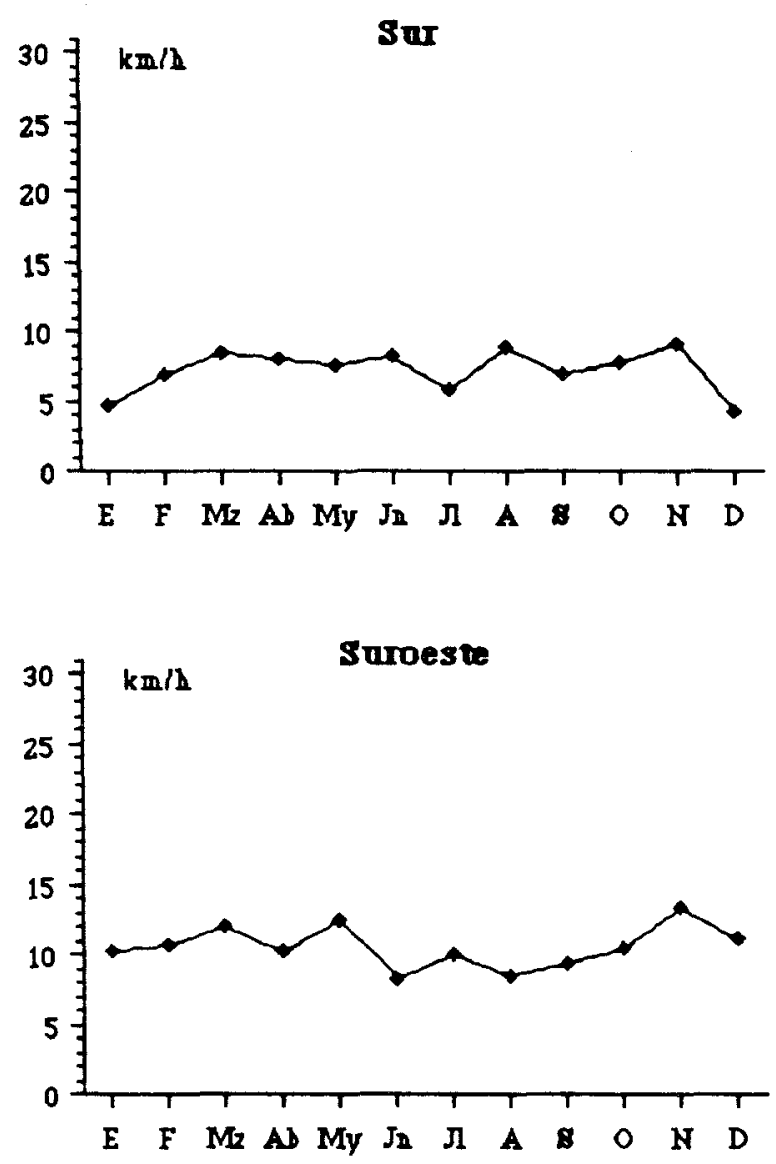

Fuente: Instituto Nacional de Meteorología. Elaboración propia.

De los 0 a los $12 \mathrm{~km} / \mathrm{h}$. la acción del viento no es desfavorable, pero a partir de entonces se multiplican sus efectos mecánicos y fisiológicos (el $63,2 \%$ al año en Zaragoza). Hasta los $20 \mathrm{~km} / \mathrm{h}$. el viento origina problemas de tipo fisiológico, entre los que se puede destacar que acelera la evapotranspiración y reseca las plantas (el 42,9\% de las ocasiones en que se observa viento a lo largo del año). Además hay que tener en cuenta que el flujo que entra en la mayor parte de estas situaciones procede del W-NW, es cierzo, que se trata de un viento frío y seco. Cuando el viento supera los $30 \mathrm{~km} / \mathrm{h}$. (el $16 \%$ al año, aproximadamente), los efectos fisiológicos se combinan con los mecánicos, lo que suele provocar considerables daños. 
Fig.4. Felocidad del riento por interralos de intensidad, en 5

0-5 KMIH

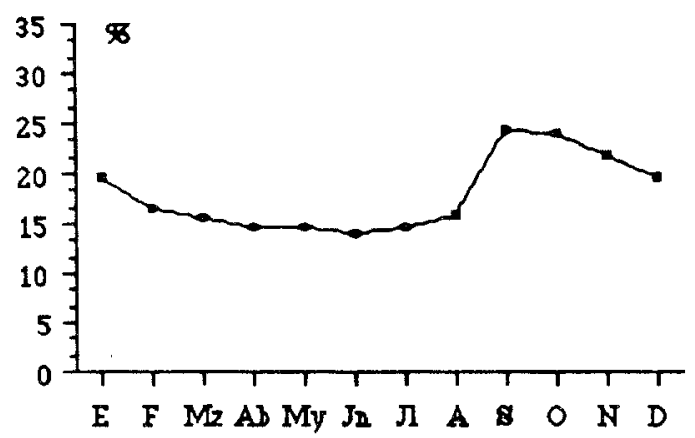

13-20 KM/H

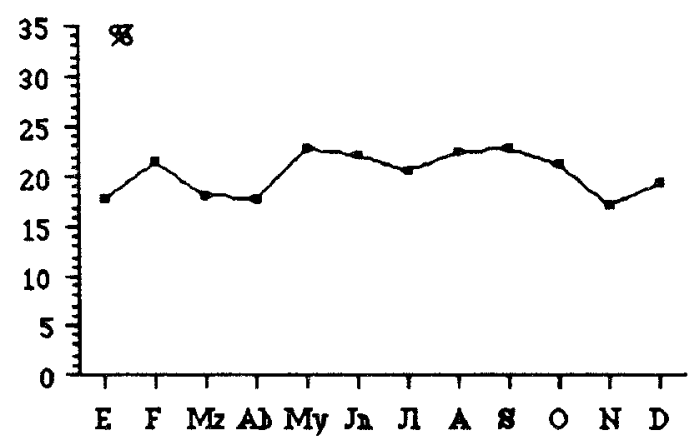

33-50 KM/H

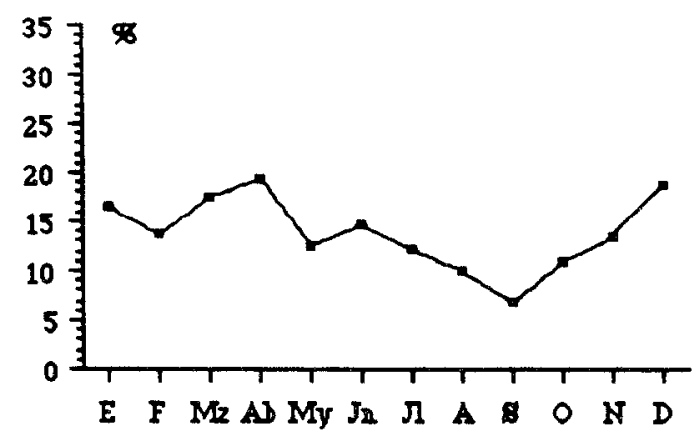

6-12 KM/H

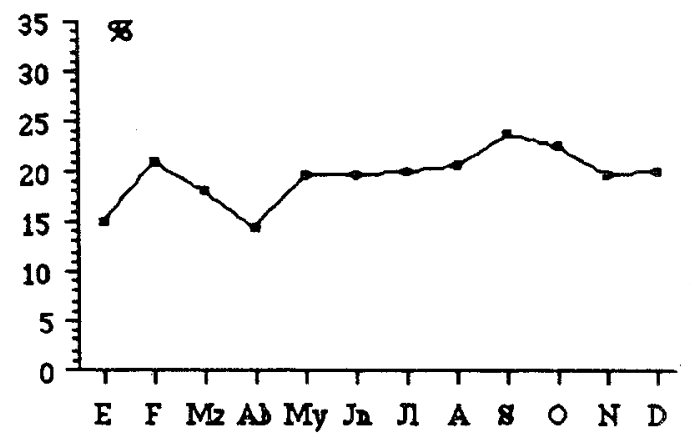

21-32 KMIH

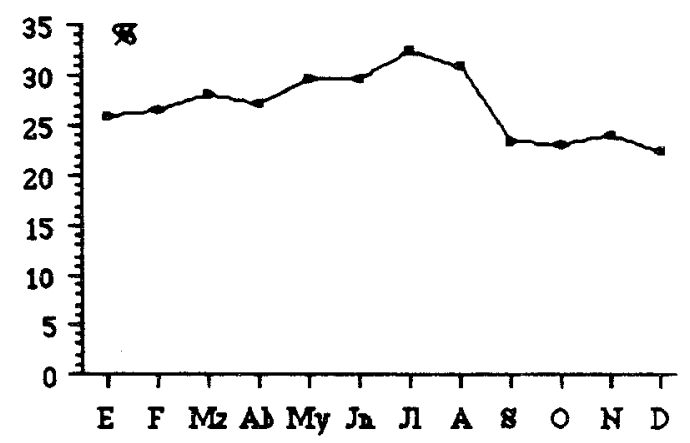

250 KMIH

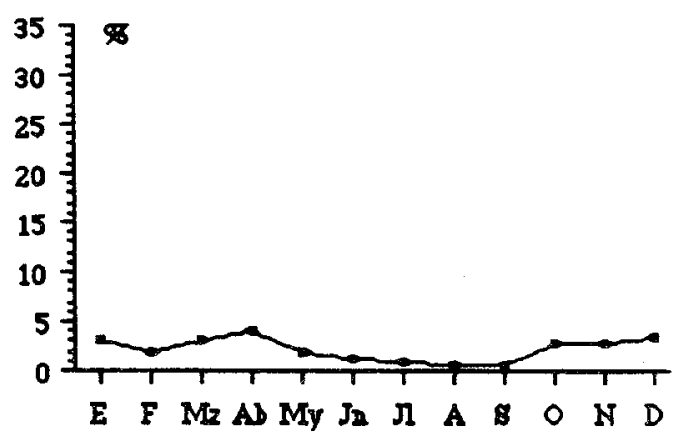

Instituto Nacional de Meteorologia. Elaboración propia.

Es habitual que el viento presente rachas máximas superiores a los 70 e incluso a los $100 \mathrm{~km} / \mathrm{h}$., como se puede apreciar en el cuadro $\mathrm{n}^{2} 1$. 
Casi la totalidad de las rachas máximas se registran con flujos del Oeste o Noroeste, con una Alta Presión en el Atlántico, y una Baja en el Mediterráneo.

Cuadro no 1. RACHAS MAXIMAS, en $\mathrm{km} / \mathrm{h}, 1957-86$

\begin{tabular}{lrrrr}
\hline & $\mathbf{k m} / \mathbf{h}$ & dirección & día & año \\
\hline ENERO & 135 & W & 20 & 1965 \\
FEBRERO & 116 & NW & 15 & 1965 \\
MARZO & 116 & WNW & 19 & 1967 \\
ABRIL & 114 & WNW & 14 & 1978 \\
MAYO & 104 & WNW & 3 & 1975 \\
JUNIO & 122 & SSW & 30 & 1965 \\
JULIO & 130 & NW & 18 & 1977 \\
AGOSTO & 96 & WNW & 15 & 1979 \\
SEPTIEMBRE & 95 & NW & 23 & 1979 \\
OCTUBRE & 108 & W & 25 y 26 & 1966 \\
NOVIEMBRE & $\mathbf{1 3 6}$ & WNW & 14 & 1979 \\
DICIEMBRE & 127 & NW & 27 & 1980 \\
\hline
\end{tabular}

Fuente: Instituto Nacional de Meteorología Elaboración propia

Vamos a analizar la situación de tiempo del 14 de noviembre de 1979, que es la que provocó los vientos más violentos de toda la treintena.

En superficie el gradiente de presión es muy fuerte sobre la Península (1008-1024 mb.), como consecuencia de la posición del Anticiclón de las Azores (1036 mb.) y de una profunda depresión sobre Europa y el Atlántico Norte ( $988 \mathrm{mb}$. en uno de los centros). Asociado a estas Borrascas un importante frente frío barre la Península en sentido Noroeste-Sureste. En la topograffa de $500 \mathrm{mb}$., las isohipsas se disponen muy apretadas, con el Jet Stream circulando a gran velocidad. Este flujo del Noroeste se encajona por efecto topográfico al entrar en el Valle del Ebro y acelera aún más su velocidad, llegando a alcanzarse a las 5 de la tarde los $136 \mathrm{~km} / \mathrm{h}$ en el Aeropuerto de Zaragoza (figura $\mathrm{n}^{2} 5$ ). 


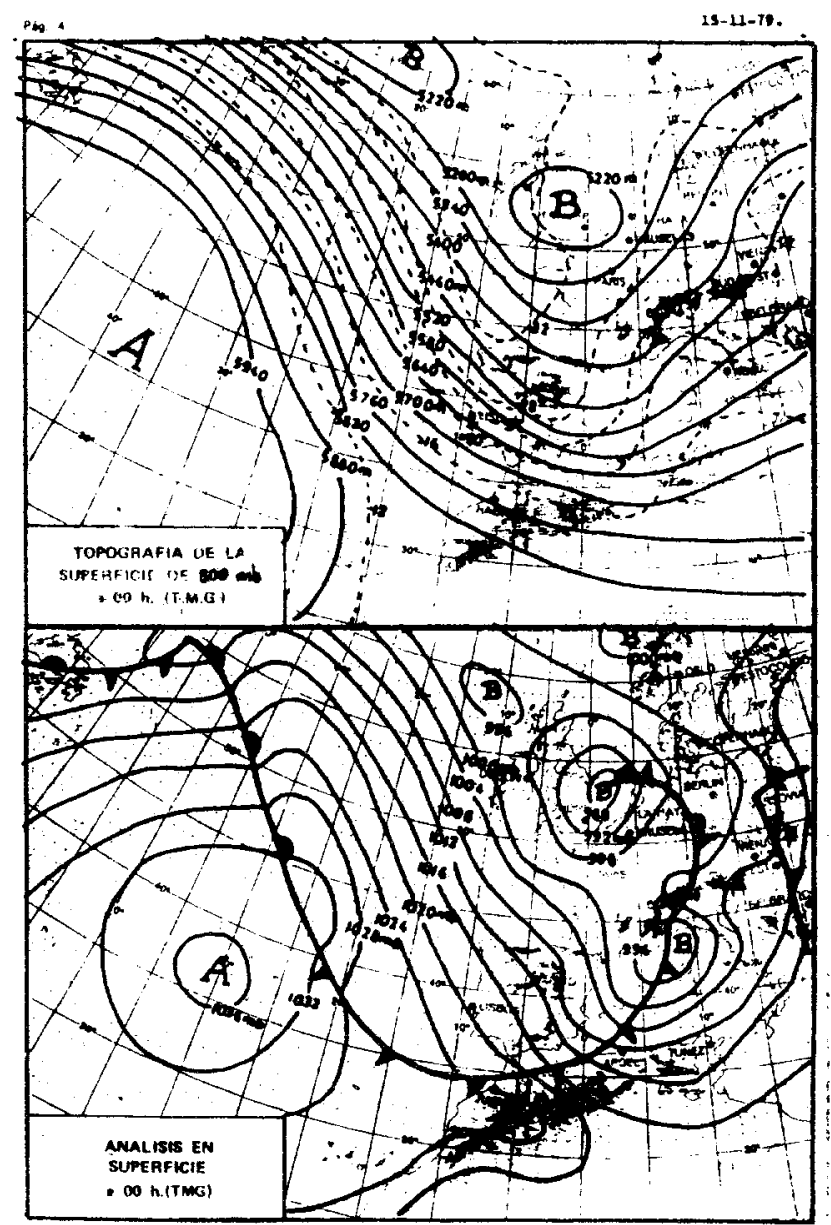

Fig. 5: Situación sinóptica día 14-11-1979

En la figura no 6 se presenta la velocidad media diaria para el periodo 1982-86, a los $20 \mathrm{~m}$. y a los $2 \mathrm{~m}$ de altura, algo inferior a la anterior y que se considera como la que afecta a las plantas. Se obtiene a partir de la transformación de los datos a $20 \mathrm{~m}$. según la siguiente expresión:

$$
\mathrm{U}_{2}=\mathrm{U}_{\mathrm{Z}}(2 / \mathrm{z})^{0,2}
$$

siendo,

$\mathrm{U}_{2}$, la velocidad del viento a $2 \mathrm{~m}$.

$\mathrm{U}_{\mathrm{Z}}$, la velocidad del viento a la altura $\mathrm{z}$. 
Fig.6. Felocidad media diaria
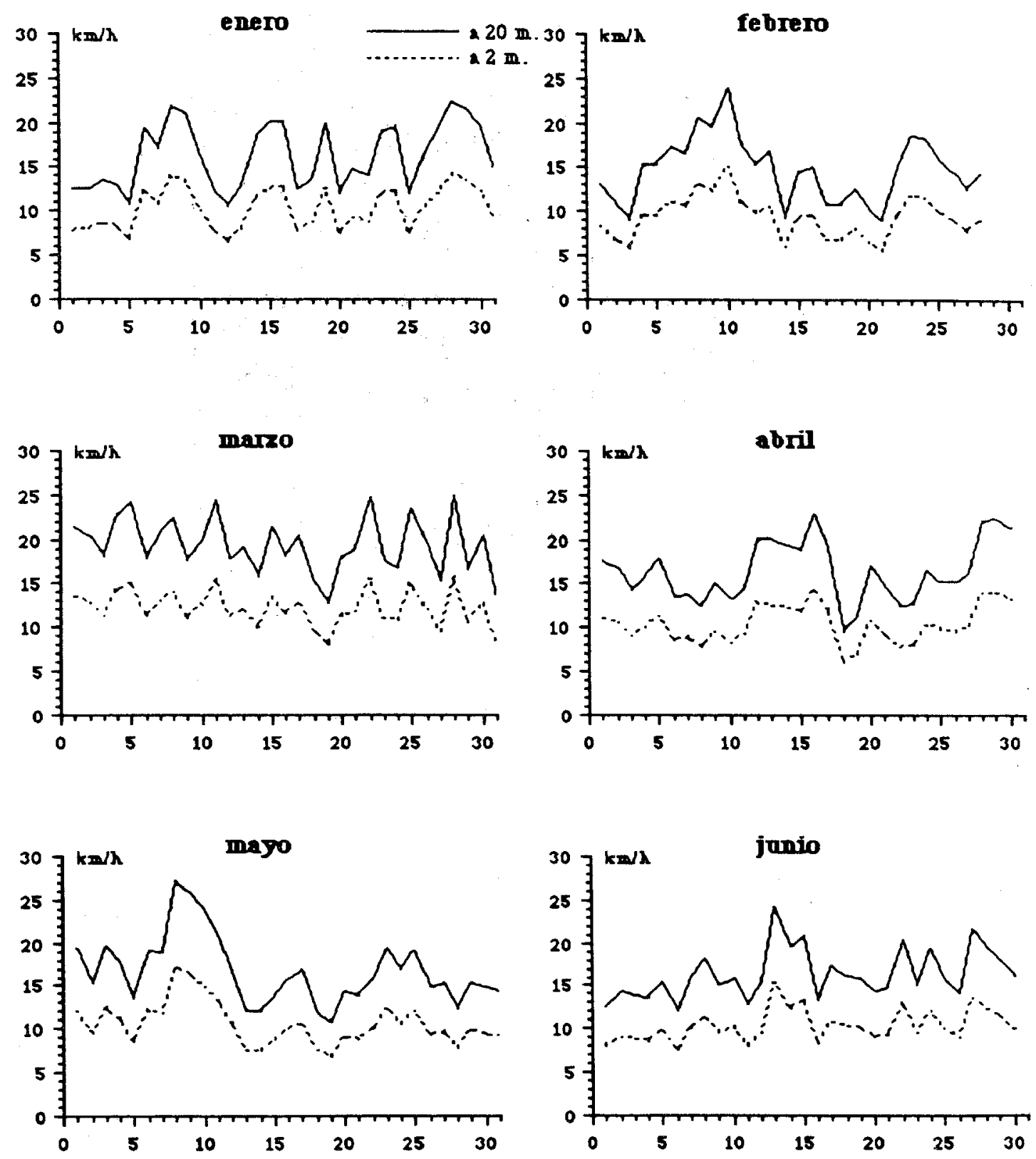

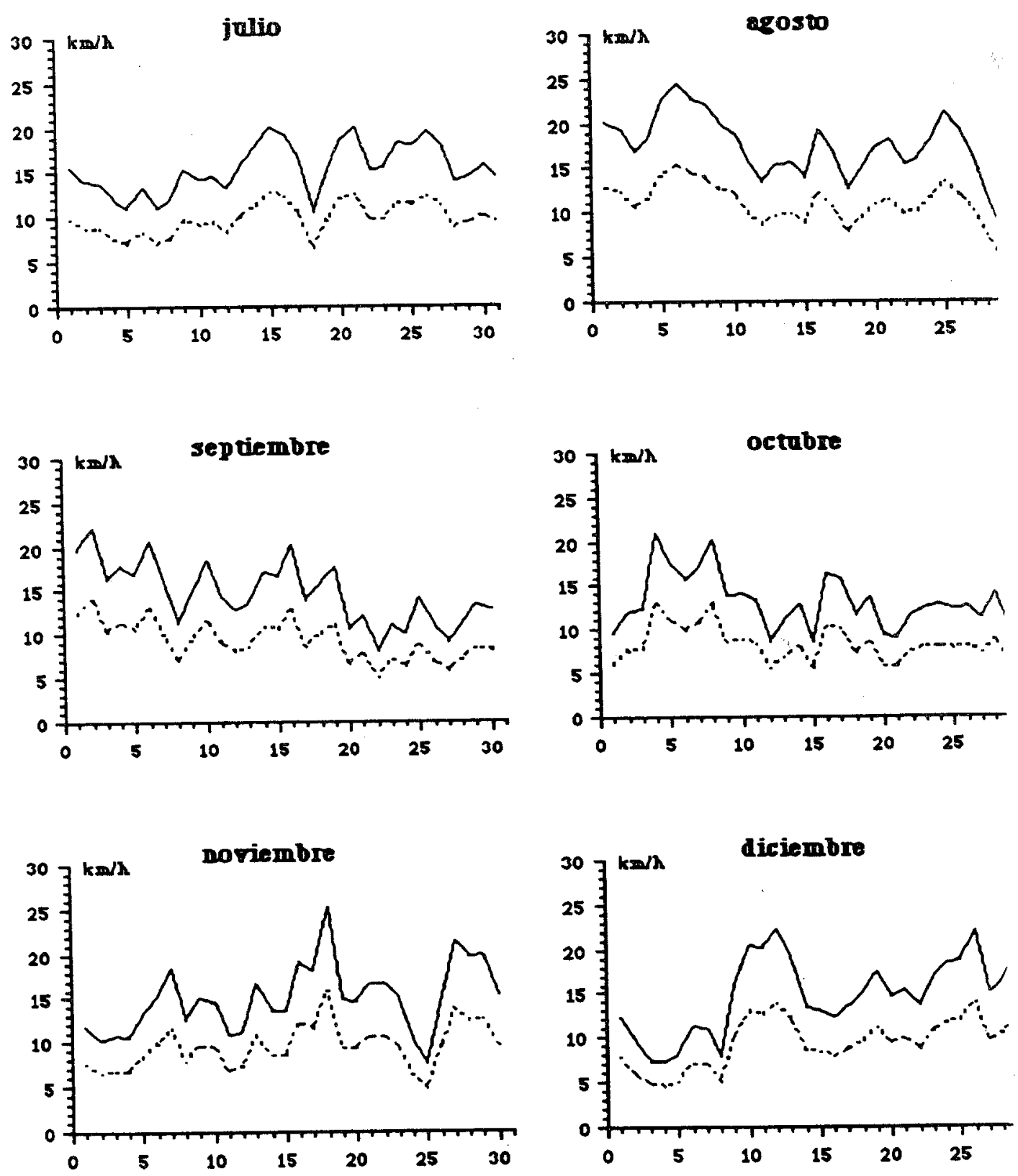

Fuente: Instituto Nacional de Meteorologia. Elaboración propia.

La figura $n^{2} 7$ presenta la velocidad media mensual del viento en 4 momentos diferentes del día, a las 0 h., a las 7,13 y 18 horas. Se puede observar que durante la primavera y el invierno el viento sopla con más velocidad en las horas centrales del día, a 
causa del calentamiento solar, mientras que durante el verano son más veloces las brisas nocturnas. Lo contrario ocurre con el viento que se observa a las $0 \mathrm{~h}$.

Fig.7. Velocidad del viento en el día

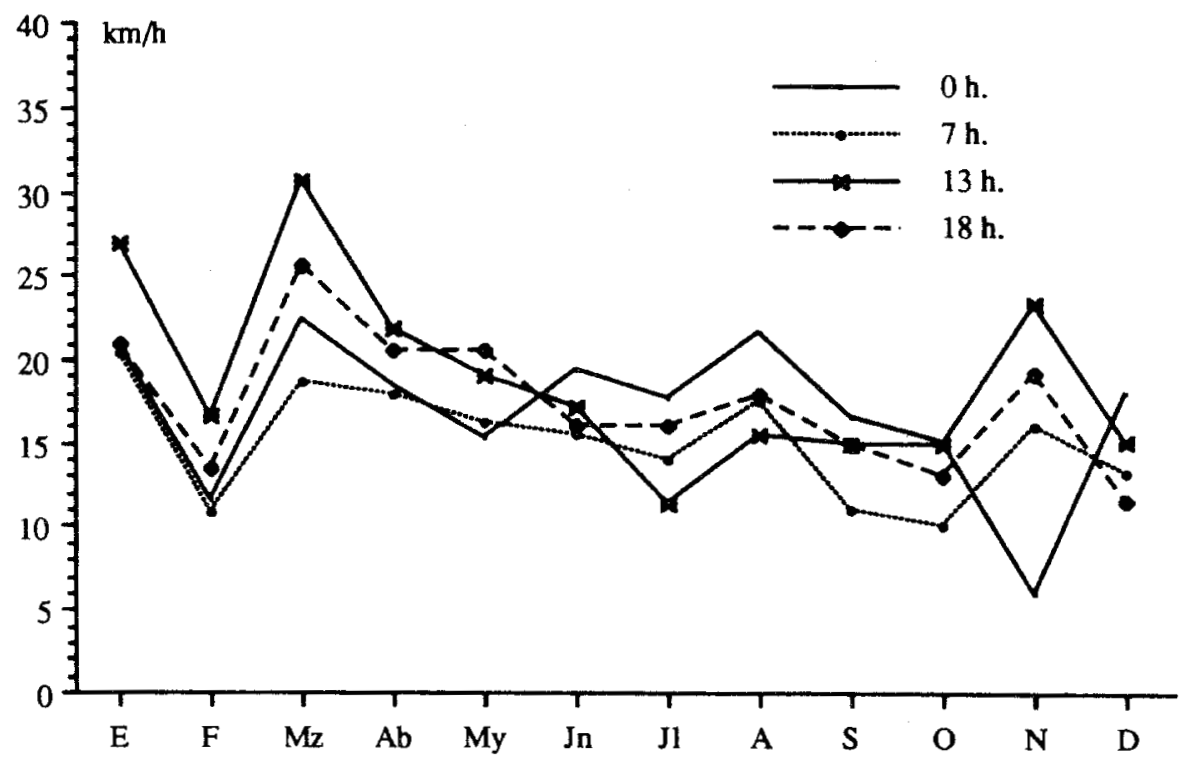

Fuente: Instituto Nacional de Meteorología Elaboración propia

La figura $\mathrm{n}^{2} 8$ refuerza lo que acabamos de comentar, a través de la relación entre el viento diurno (el valor medio entre los observados a las 7,13 y $18 \mathrm{~h}$.) y el nocturno (18 h.).

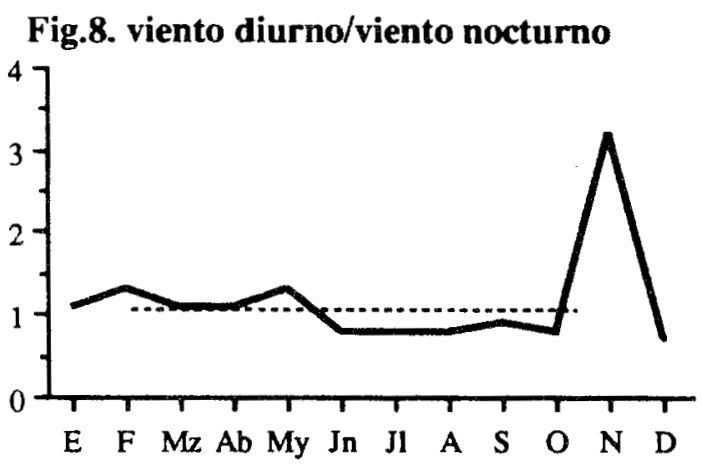

Fuente: Instituto Nacional de Meteorología Elaboración propia 


\section{CONCLUSIONES}

-El viento sopla de forma continuada e intensa en Zaragoza. Las calmas suponen un $13,5 \%$ anual. Su velocidad media es de $19 \mathrm{~km} / \mathrm{h}$, de dirección dominante $\mathrm{NW}$ y $\mathrm{SE}$, cierzo y bochorno. La velocidad es particularmente alta durante el invierno y la primavera.

-En un $60 \%$ de las ocasiones el viento se observa con una velocidad superior a los $12 \mathrm{~km} / \mathrm{h}$, velocidad umbral a partir de la que los efectos comienzan a ser más perjudiciales que beneficiosos. Un $43 \%$ de las ocasiones sopla con una velocidad superior a $\operatorname{los} 20 \mathrm{~km} / \mathrm{h}$ y en un $16 \%$ supera los $30 \mathrm{Km} / \mathrm{h}$. Se registran rachas máximas con velocidades de más de $100 \mathrm{~km} / \mathrm{h}$.

-El viento presenta, en Zaragoza, importantes efectos perjudiciales fisiológicos y mecánicos.

\section{BIBLIOGRAFIA}

ACASO LIRIA, A. ; CUADRAT PRATS, J.M. (1981). "El clima" en VV.AA. Geografía de Aragón, Ed. Guara. tomo 1, 93-140.

BIEL LUCEA, A. (1952). El viento en Zaragoza Servicio Meteorológico Nacional, Serie D, $\mathrm{n}^{2} 12,62 \mathrm{p}$.

BIEL LUCEA, A; GARCIA DE PEDRAZA, L. (1962). El clima de Zaragoza y ensayo climático para el Valle del Ebro, Servicio Meteorológico Nacional, Serie A, n² 36, 56 p.

BIEL LUCEA, A. "Vientos del Valle medio del Ebro", Boletín climatológico del Servicio Meteorológico Nacional.

FRUTOS MEJIAS, L.M. (1976). Estudio gegráfico del Campo de Zaragoza , Institución "Fernando el Católico". 353 p.

HERNANDEZ NAVARRO, M.L. (en prensa) Agroclimatología de Zaragoza, Institución "Fernando el Catolico", $150 \mathrm{p}$.

ORGANIZACION METEOROLOGICA MUNDIAL. (1970). Guía de práctticas climatologicas O.M.M. $n^{\circ} 100$. TP 44. 